\title{
Supplementary Materials for Smoothed Lexis Diagrams with Applications to Lung and Breast Cancer Trends in Taiwan
}

Li-Chu Chien, ${ }^{\mathrm{a}}{ }^{\S}$ Yuh-Jenn Wu, ${ }^{b \S}$ Chao A. Hsiung, ${ }^{\mathrm{a}}$ Lu-Hai Wang ${ }^{\mathrm{c}}$ and I-Shou Chang ${ }^{\mathrm{a}, \mathrm{d} * \dagger}$

${ }^{a}$ Division of Biostatistics and Bioinformatics, Institute of Population Health Sciences, National Health Research Institutes, Taiwan

${ }^{\mathrm{b}}$ Department of Applied Mathematics, Chung Yuan Christian University, Taiwan ${ }^{\mathrm{c}}$ Institute of Molecular and Genomic Medicine, National Health Research Institutes, Taiwan

${ }^{\mathrm{d}}$ National Institute of Cancer Research, National Health Research Institutes, Taiwan

${ }^{*}$ correspondence to: I-Shou Chang, National Institute of Cancer Research, National Health Research Institutes, Taiwan.

${ }^{\dagger}$ E-mail: ischang@nhri.org.tw

${ }^{\S}$ These two authors contributed equally, and both are co-first authors.

March 17, 2015 


\section{APPENDIX A. ALGORITHM (MHRJ FOR THE POSTERIOR)}

Let $B_{(n)}=\left\{\left(n, a_{0,0}, \ldots, a_{n, n}\right) \mid\left(a_{0,0}, \ldots, a_{n, n}\right) \in \tilde{B}_{n}\right\}$. Let $\theta^{(t)}=\left(n, a_{0,0}, \ldots, a_{n, n}\right) \in B_{(n)}$ be the current state. We describe the transition from $\theta^{(t)} \in B_{(n)}$ to a new point $\theta^{(t+1)}$ by the following algorithms.

Randomly select one of three types of moves $H, H^{+}$, or $H^{-}$. Here $H$ is a transition of element in $B_{(n)}, H^{+}$a transition of element from $B_{(n)}$ to $B_{(n+1)}$, and $H^{-}$a transition of element from $B_{(n)}$ to $B_{(n-1)}$, respectively. Denote by $E_{H}^{n}, E_{H^{+}}^{n}$ and $E_{H^{-}}^{n}$ the probabilities of selecting the three different types of moves $-H$, $H^{+}$, and $H^{-}$- when the current state of the Markov chain is in $B_{(n)}$. We set $E_{H^{-}}^{2}=E_{H^{+}}^{n_{0}}=0$. Consider $E_{H}^{n}=1-E_{H^{+}}^{n}-E_{H^{-}}^{n}, E_{H^{+}}^{n}=c \min \{1, p(n+1) / p(n)\}$ and $E_{H^{-}}^{n}=c \min \{1, p(n-1) / p(n)\}$, where $p$ is as given in Section 2.2 and $c$ is a sample parameter as described in Section 3. We note that in the following, $v$ denotes the posterior density introduced in Section 2.1.

If the move of type $H^{+}$is selected, then

1. randomly choose $g$ and $h$ from $\{1, \ldots, n\}$;

2. generate $W_{0} \sim \operatorname{Uniform}\left(M_{1}, l_{1}\right)$ and $W_{n+1} \sim \operatorname{Uniform}\left(M_{1}, l_{2}\right)$;

3. generate $W_{i} \sim \operatorname{Uniform}\left(M_{1}, M_{2}\right)$ for $i=1, \ldots, n$;

4. generate $V_{0} \sim \operatorname{Uniform}\left(M_{1}, l_{3}\right)$ and $V_{n} \sim \operatorname{Uniform}\left(M_{1}, l_{4}\right)$;

5. generate $V_{j} \sim \operatorname{Uniform}\left(M_{1}, M_{2}\right)$ for $j=1, \ldots, n-1$;

6. let $s^{(t)}=\left(n+1, \mathbf{a}_{0}, \ldots, \mathbf{a}_{g-1}, \breve{a}, \mathbf{a}_{g}, \ldots, \mathbf{a}_{n}\right) \in B_{(n+1)}$ with $\breve{\mathbf{a}}=\left(W_{0}, \ldots, W_{n+1}\right)$ and $\mathbf{a}_{j}=\left(a_{0, j}, \ldots, a_{h-1, j}, V_{j}, a_{h, j}, \ldots, a_{n, j}\right)$ for $j=0, \ldots, n$; 
7. set the next state

$$
\theta^{(t+1)}=\left\{\begin{array}{l}
s^{(t)}, \text { with prob. } \rho=\min \left\{1, \frac{v\left(s^{(t)}\right) \times p(n) \times \prod_{d=1}^{4}\left(l_{d}-M_{1}\right) \times\left(M_{2}-M_{1}\right)^{2 n-1}}{v\left(\theta^{(t)}\right) \times p(n+1)}\right\}, \\
\theta^{(t)}, \text { o.w. }
\end{array}\right.
$$

If the move of type $\mathrm{H}^{-}$is selected, then

1. randomly choose $g$ and $h$ from $\{1, \ldots, n-1\}$;

2. let $s^{(t)}=\left(n-1, \mathbf{a}_{0}, \ldots, \mathbf{a}_{g-1}, \mathbf{a}_{g+1}, \ldots, \mathbf{a}_{n}\right) \in B_{(n-1)}$ with $\mathbf{a}_{j}=\left(a_{0, j}, \ldots, a_{h-1, j}, a_{h+1, j}, \ldots a_{n, j}\right)$ for $j=0, \ldots, g-1, g+1, \ldots, n$;

3. set the next state

$$
\theta^{(t+1)}=\left\{\begin{array}{l}
s^{(t)}, \text { with prob. } \rho=\min \left\{1, \frac{v\left(s^{(t)}\right) \times p(n)}{v\left(\theta^{(t)}\right) \times p(n-1) \times \prod_{d=1}^{4}\left(l_{d}-M_{1}\right) \times\left(M_{2}-M_{1}\right)^{2 n-3}}\right\}, \\
\theta^{(t)} \text {, o.w. }
\end{array}\right.
$$

If the move of type $H$ is selected, then randomly choose a updating procedure from the following.

i) Update $a_{0,0}$.

1. generate $\breve{a}_{0,0} \sim q_{1}$;

2. let $s^{(t)}$ be the vector $\theta^{(t)}$ with $a_{0,0}$ replaced by $\breve{a}_{0,0}$;

3. set the next state

$$
\theta^{(t+1)}=\left\{\begin{array}{l}
s^{(t)}, \text { with prob. } \rho=\min \left\{1, \frac{v\left(s^{(t)}\right)}{v\left(\theta^{(t)}\right)}\right\} \\
\theta^{(t)} \text {, o.w. }
\end{array}\right.
$$


ii) Update $a_{0, n}$.

1. generate $\breve{a}_{0, n} \sim q_{2}$;

2 . let $s^{(t)}$ be the vector $\theta^{(t)}$ with $a_{0, n}$ replaced by $\breve{a}_{0, n}$;

3. set the next state

$$
\theta^{(t+1)}=\left\{\begin{array}{l}
s^{(t)}, \text { with prob. } \rho=\min \left\{1, \frac{v\left(s^{(t)}\right)}{v\left(\theta^{(t)}\right)}\right\}, \\
\theta^{(t)}, \text { o.w. }
\end{array}\right.
$$

iii) Update $a_{n, 0}$.

1. generate $\breve{a}_{n, 0} \sim q_{3}$;

2. let $s^{(t)}$ be the vector $\theta^{(t)}$ with $a_{n, 0}$ replaced by $\breve{a}_{n, 0}$;

3. set the next state

$$
\theta^{(t+1)}=\left\{\begin{array}{l}
s^{(t)}, \text { with prob. } \rho=\min \left\{1, \frac{v\left(s^{(t)}\right)}{v\left(\theta^{(t)}\right)}\right\}, \\
\theta^{(t)}, \text { o.w. }
\end{array}\right.
$$

iv) Update $a_{n, n}$.

1. generate $\breve{a}_{n, n} \sim q_{4}$;

2. let $s^{(t)}$ be the vector $\theta^{(t)}$ with $a_{n, n}$ replaced by $\breve{a}_{n, n}$;

3. set the next state

$$
\theta^{(t+1)}=\left\{\begin{array}{l}
s^{(t)}, \text { with prob. } \rho=\min \left\{1, \frac{v\left(s^{(t)}\right)}{v\left(\theta^{(t)}\right)}\right\}, \\
\theta^{(t)} \text {, o.w. }
\end{array}\right.
$$

v) Update $a_{0, j}$ for $j=1, \ldots, n-1$.

1. generate $\breve{a}_{0, j} \sim \operatorname{Uniform}\left(M_{1}, l_{1}\right)$ for $j=1, \ldots, n-1$; 
2. let $s^{(t)}$ be the vector $\theta^{(t)}$ with $a_{0,1}, \ldots, a_{0, n-1}$ replaced by $\breve{a}_{0,1}, \ldots, \breve{a}_{0, n-1}$, respectively;

3. set the next state

$$
\theta^{(t+1)}=\left\{\begin{array}{l}
s^{(t)}, \text { with prob. } \rho=\min \left\{1, \frac{v\left(s^{(t)}\right)}{v\left(\theta^{(t)}\right)}\right\}, \\
\theta^{(t)} \text {, o.w. }
\end{array}\right.
$$

vi) Update $a_{n, j}$ for $j=1, \ldots, n-1$.

1. generate $\breve{a}_{n, j} \sim \operatorname{Uniform}\left(M_{1}, l_{2}\right)$ for $j=1, \ldots, n-1$;

2. let $s^{(t)}$ be the vector $\theta^{(t)}$ with $a_{n, 1}, \ldots, a_{n, n-1}$ replaced by $\breve{a}_{n, 1}, \ldots, \breve{a}_{n, n-1}$, respectively;

3. set the next state

$$
\theta^{(t+1)}=\left\{\begin{array}{l}
s^{(t)}, \text { with prob. } \rho=\min \left\{1, \frac{v\left(s^{(t)}\right)}{v\left(\theta^{(t)}\right)}\right\}, \\
\theta^{(t)}, \text { o.w. }
\end{array}\right.
$$

vii) Update $a_{i, 0}$ for $i=1, \ldots, n-1$.

1. generate $\breve{a}_{i, 0} \sim \operatorname{Uniform}\left(M_{1}, l_{3}\right)$ for $i=1, \ldots, n-1$;

2. let $s^{(t)}$ be the vector $\theta^{(t)}$ with $a_{1,0}, \ldots, a_{n-1,0}$ replaced by $\breve{a}_{1,0}, \ldots, \breve{a}_{n-1,0}$, respectively;

3. set the next state

$$
\theta^{(t+1)}=\left\{\begin{array}{l}
s^{(t)}, \text { with prob. } \rho=\min \left\{1, \frac{v\left(s^{(t)}\right)}{v\left(\theta^{(t)}\right)}\right\}, \\
\theta^{(t)} \text {, o.w. }
\end{array}\right.
$$

viii) Update $a_{i, n}$ for $i=1, \ldots, n-1$.

1. generate $\breve{a}_{i, n} \sim \operatorname{Uniform}\left(M_{1}, l_{4}\right)$ for $i=1, \ldots, n-1$;

2 . let $s^{(t)}$ be the vector $\theta^{(t)}$ with $a_{1, n}, \ldots, a_{n-1, n}$ replaced by $\breve{a}_{1, n}, \ldots, \breve{a}_{n-1, n}$, 
respectively;

3. set the next state

$$
\theta^{(t+1)}=\left\{\begin{array}{l}
s^{(t)}, \text { with prob. } \rho=\min \left\{1, \frac{v\left(s^{(t)}\right)}{v\left(\theta^{(t)}\right)}\right\}, \\
\theta^{(t)}, \text { o.w. }
\end{array}\right.
$$

ix) Update $a_{1, j}, \ldots, a_{n-1, j}$ for any $j=1, \ldots, n-1$.

1. randomly choose $g$ from $\{1, \ldots, n-1\}$;

2. generate $\breve{a}_{i, g} \sim \operatorname{Uniform}\left(M_{1}, M_{2}\right)$ for $i=1, \ldots, n-1$;

2. let $s^{(t)}$ be the vector $\theta^{(t)}$ with $a_{1, g}, \ldots, a_{n-1, g}$ replaced by $\breve{a}_{1, g}, \ldots, \breve{a}_{n-1, g}$, respectively;

3. set the next state

$$
\theta^{(t+1)}=\left\{\begin{array}{l}
s^{(t)}, \text { with prob. } \rho=\min \left\{1, \frac{v\left(s^{(t)}\right)}{v\left(\theta^{(t)}\right)}\right\}, \\
\theta^{(t)}, \text { o.w. }
\end{array}\right.
$$




\section{APPENDIX B.}

For the FIBC study, we consider cases with ICD-9-CM codes 1740-1746, 1748, and 1749, and morphological code 85003 (infiltrating duct carcinoma). For lung cancer, we consider cases with ICD-9-CM codes 1620, 1622-1625, 1628, and 1629. For lung ADC, the morphological codes include 81403, 81413, 81433, 82113, 82503, 82513, 82553, 82603, 83103, 83233, 84403, 84803, 84813, 84903, and 85003. For the lung SCC, the morphological codes include 80523, 80703, 80713, 80723, 80733, 80743, 80763, 80823, 80833, and 80843 . 


\section{APPENDIX C.}

Tables C1, C2, C3, and C4: These tables show characteristics of the MCMC for each analysis. Each row describes the characters of one Bayesian analysis. The first column lists the four sets of priors used for the analysis, given in Section 3. Columns 2 and 3 give, respectively, the mean and maximum of $\hat{F} \times 10^{5}$. Columns 4, 5, and 6 give the Gelman-Rubin statistic monitoring chain convergence, the number of iterations, and the CUP time for this chain, respectively. Column 7 gives the posterior predictive $p$-value.

Tables C1-1, C2-1, C3-1, and C4-1: These tables report sensitivity analysis regarding Tables C1, C2, C3, and C4, respectively. For example, Table C1-1 reports the difference between any two Bayesian analyses in Table C1, using the criterion $\mathrm{T}=\max \left\{\left|\hat{F}_{\text {prior } i}\left(x_{k}, y_{k}\right)-\hat{F}_{\text {prior } j}\left(x_{k}, y_{k}\right)\right| \mid k=1, \ldots, 1020\right\} \times 10^{5}$, the largest difference happens between set of priors 2 and 3 and has the value of 7.312.

Table C1. FIBC

\begin{tabular}{ccccccc}
\hline $\begin{array}{c}\text { Set } \\
\text { of priors }\end{array}$ & Mean & Max & $\hat{R}$ & Iterations & $\begin{array}{c}\text { Time } \\
\text { (min.) }\end{array}$ & $P$-value \\
\hline 1 & 58.33 & 135.7 & 1.039 & 200,000 & 7 & 0.600 \\
2 & 58.32 & 133.1 & 1.042 & 200,000 & 7 & 0.508 \\
3 & 58.48 & 136.2 & 1.088 & 400,000 & 14 & 0.500 \\
4 & 58.32 & 136.1 & 1.008 & 800,000 & 27 & 0.620 \\
\hline
\end{tabular}

Table C1-1. Sensitivity analysis regarding Table C1

\begin{tabular}{ccccccc}
\hline \hline Set of priors & $(1,2)$ & $(1,3)$ & $(1,4)$ & $(2,3)$ & $(2,4)$ & $(3,4)$ \\
$\mathrm{T}$ & 2.580 & 6.738 & 1.352 & 7.312 & 3.045 & 7.123 \\
\hline
\end{tabular}


Table C2. FADC

\begin{tabular}{ccccccc}
\hline $\begin{array}{c}\text { Set } \\
\text { of priors }\end{array}$ & Mean & Max & $\hat{R}$ & Iterations & $\begin{array}{c}\text { Time } \\
\text { (min.) }\end{array}$ & $P$-value \\
\hline 1 & 36.79 & 167.0 & 1.015 & 200,000 & 7 & 0.385 \\
2 & 36.79 & 167.0 & 1.015 & 200,000 & 6 & 0.418 \\
3 & 36.79 & 167.8 & 1.013 & 200,000 & 7 & 0.385 \\
4 & 36.79 & 167.1 & 1.003 & 200,000 & 7 & 0.401 \\
\hline
\end{tabular}

Table C2-1. Sensitivity analysis regarding Table C2

\begin{tabular}{ccccccc}
\hline \hline Set of priors & $(1,2)$ & $(1,3)$ & $(1,4)$ & $(2,3)$ & $(2,4)$ & $(3,4)$ \\
$\mathrm{T}$ & $3.385 \times 10^{-4}$ & 1.081 & 0.727 & 1.081 & 0.727 & 0.354 \\
\hline
\end{tabular}

Table C3. MADC

\begin{tabular}{ccccccc}
\hline $\begin{array}{c}\text { Set } \\
\text { of priors }\end{array}$ & Mean & Max & $\hat{R}$ & Iterations & $\begin{array}{c}\text { Time } \\
\text { (min.) }\end{array}$ & $P$-value \\
\hline 1 & 47.50 & 207.4 & 1.002 & 200,000 & 7 & 0.306 \\
2 & 47.50 & 207.4 & 1.002 & 200,000 & 6 & 0.345 \\
3 & 47.47 & 207.4 & 1.016 & 200,000 & 7 & 0.334 \\
4 & 47.47 & 207.4 & 1.016 & 200,000 & 7 & 0.319 \\
\hline
\end{tabular}

Table C3-1. Sensitivity analysis regarding Table C3

\begin{tabular}{ccccccc}
\hline \hline Set of priors & $(1,2)$ & $(1,3)$ & $(1,4)$ & $(2,3)$ & $(2,4)$ & $(3,4)$ \\
$\mathrm{T}$ & 0.001 & 0.394 & 0.394 & 0.393 & 0.393 & $7.575 \times 10^{-4}$ \\
\hline
\end{tabular}

Table C4. MSCC

\begin{tabular}{ccccccc}
\hline \hline $\begin{array}{c}\text { Set } \\
\text { of priors }\end{array}$ & Mean & Max & $\hat{R}$ & Iterations & $\begin{array}{c}\text { Time } \\
\text { (min.) }\end{array}$ & $P$-value \\
\hline 1 & 50.23 & 220.1 & 1.096 & $1,600,000$ & 53 & 0.029 \\
2 & 50.17 & 219.2 & 1.030 & $6,400,000$ & 218 & 0.027 \\
3 & 46.07 & 153.5 & 1.013 & 200,000 & 6 & 0.195 \\
4 & 46.07 & 153.5 & 1.013 & 200,000 & 6 & 0.183 \\
\hline
\end{tabular}


Table C4-1. Sensitivity analysis regarding Table C4

\begin{tabular}{ccccccc}
\hline \hline Set of priors & $(1,2)$ & $(1,3)$ & $(1,4)$ & $(2,3)$ & $(2,4)$ & $(3,4)$ \\
$\mathrm{T}$ & 4.897 & 68.50 & 68.50 & 67.51 & 67.51 & $5.248 \times 10^{-4}$ \\
\hline
\end{tabular}




\section{APPENDIX D.}

a. FIBC

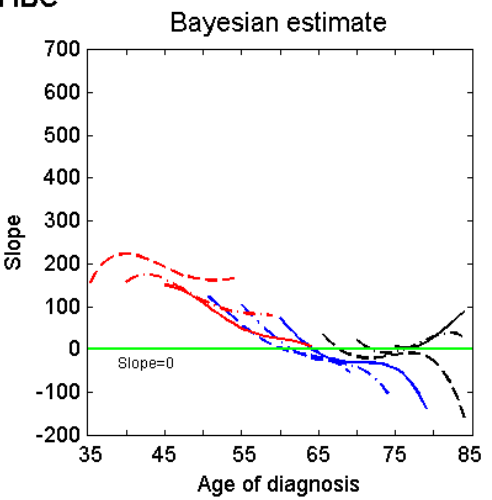

c. MADC

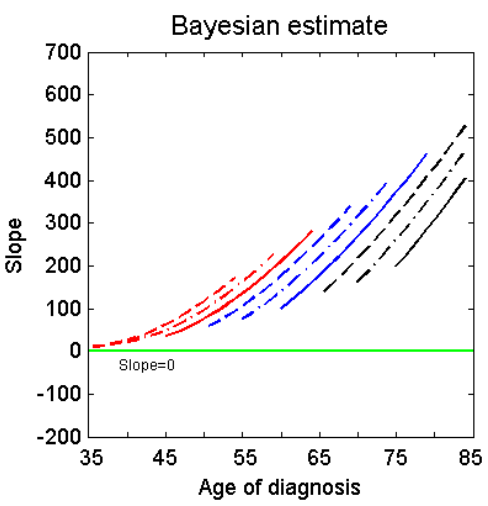

b. FADC
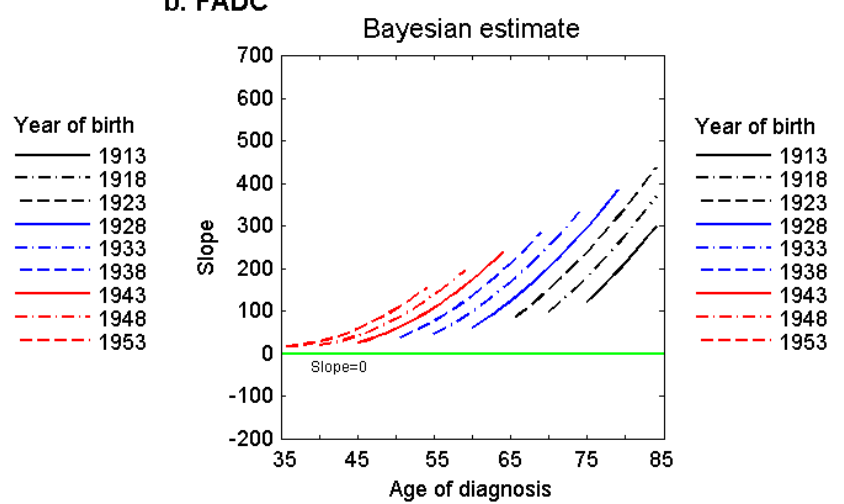

d. MSCC

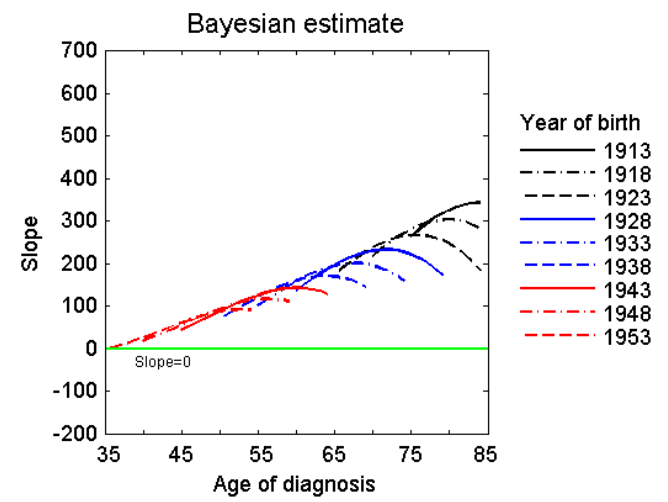

Figure D1. Derivative (rate of change) of age-specific rates by year of birth, plotted in Figure 3. Light green horizontal lines have slopes of zero. 
a. FIBC

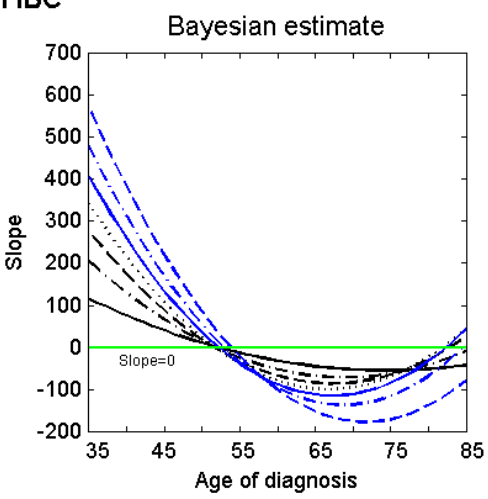

c. MADC

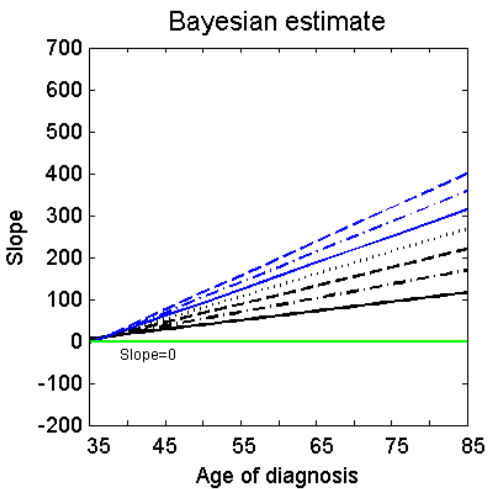

b. FADC

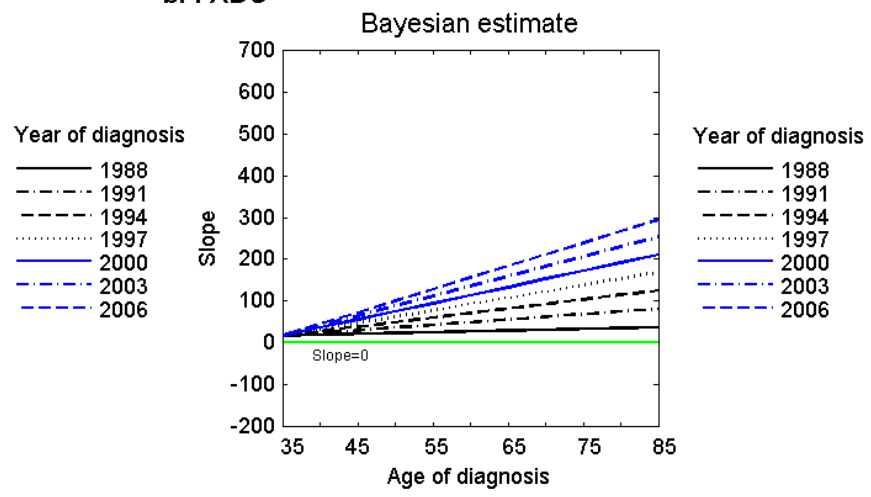

d. MSCC

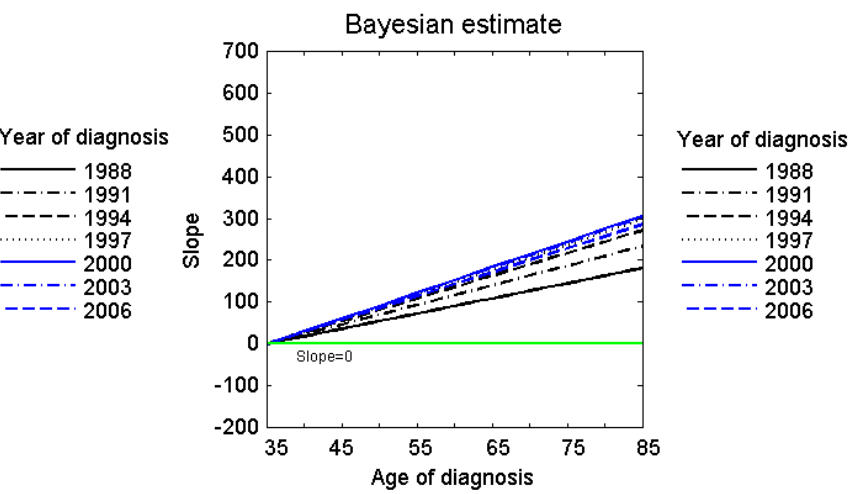

Figure D2. Derivative (rate of change) of age-specific rates by year of diagnosis, plotted in Figure 4. Light green horizontal lines have slopes of zero. 

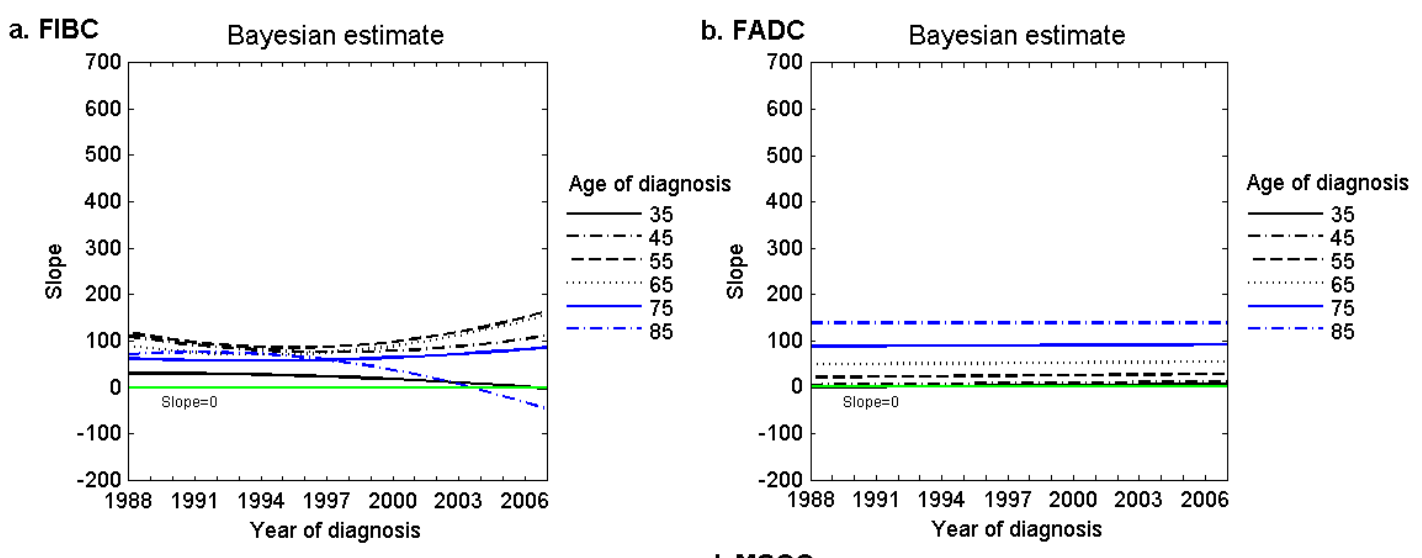

c. MADC

Bayesian estimate
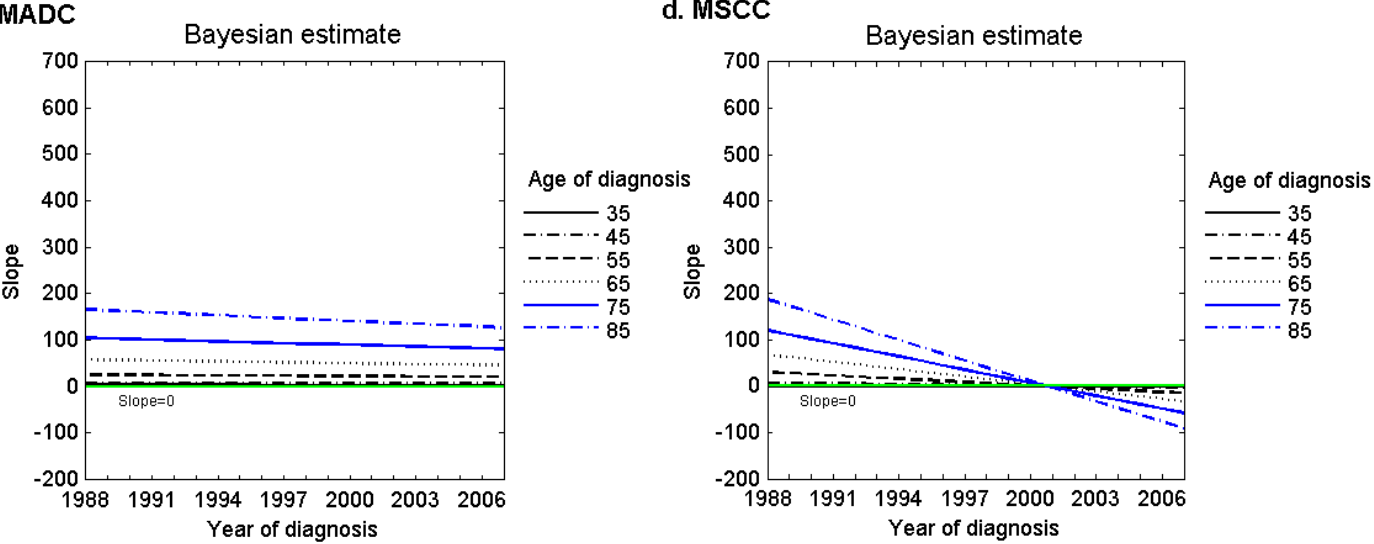

Figure D3. Derivative (rate of change) of year-specific rates by age of

diagnosis, plotted in Figure 5. Light green horizontal lines have slopes of zero. 
a. FIBC

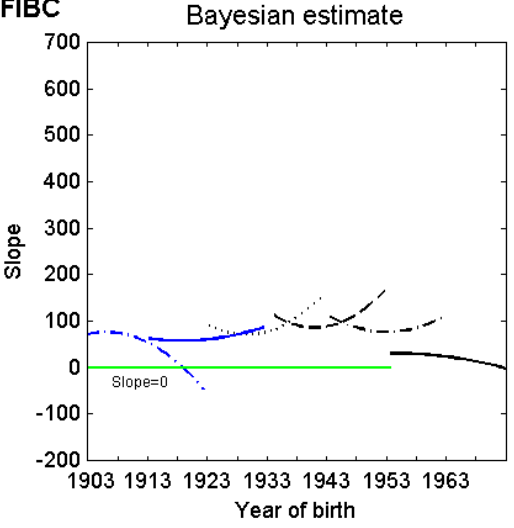

c. MADC

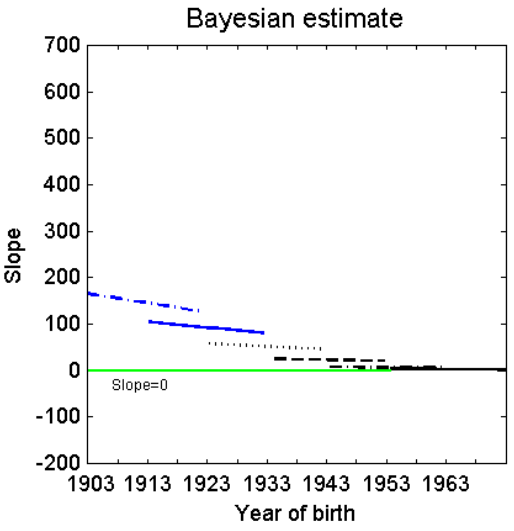

b. FADC Bayesian estimate

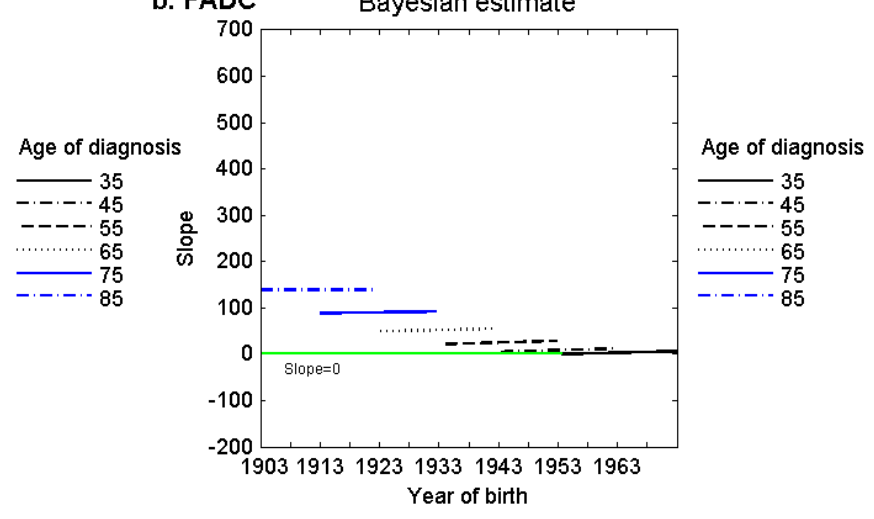

d. MSCC

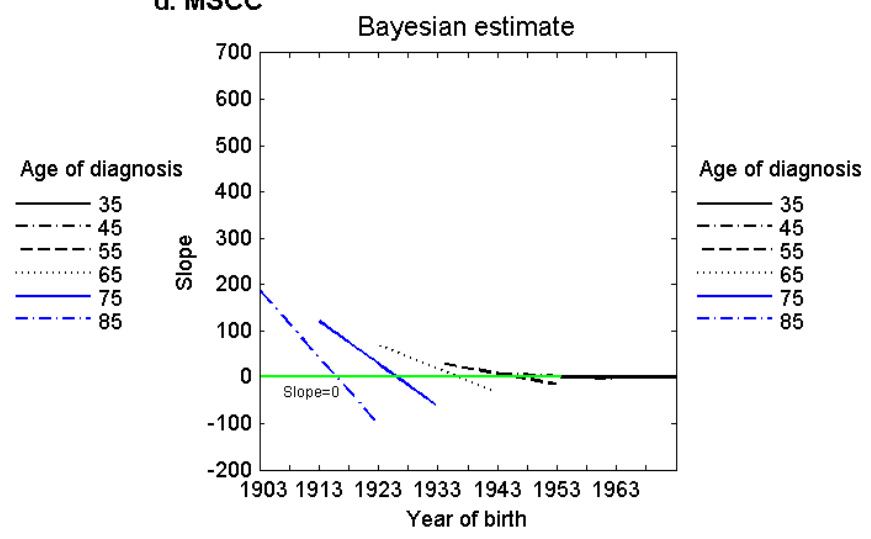

Figure D4. Derivative (rate of change) of cohort-specific rates by age of diagnosis, plotted in Figure 6. Light green horizontal lines have slopes of zero. 


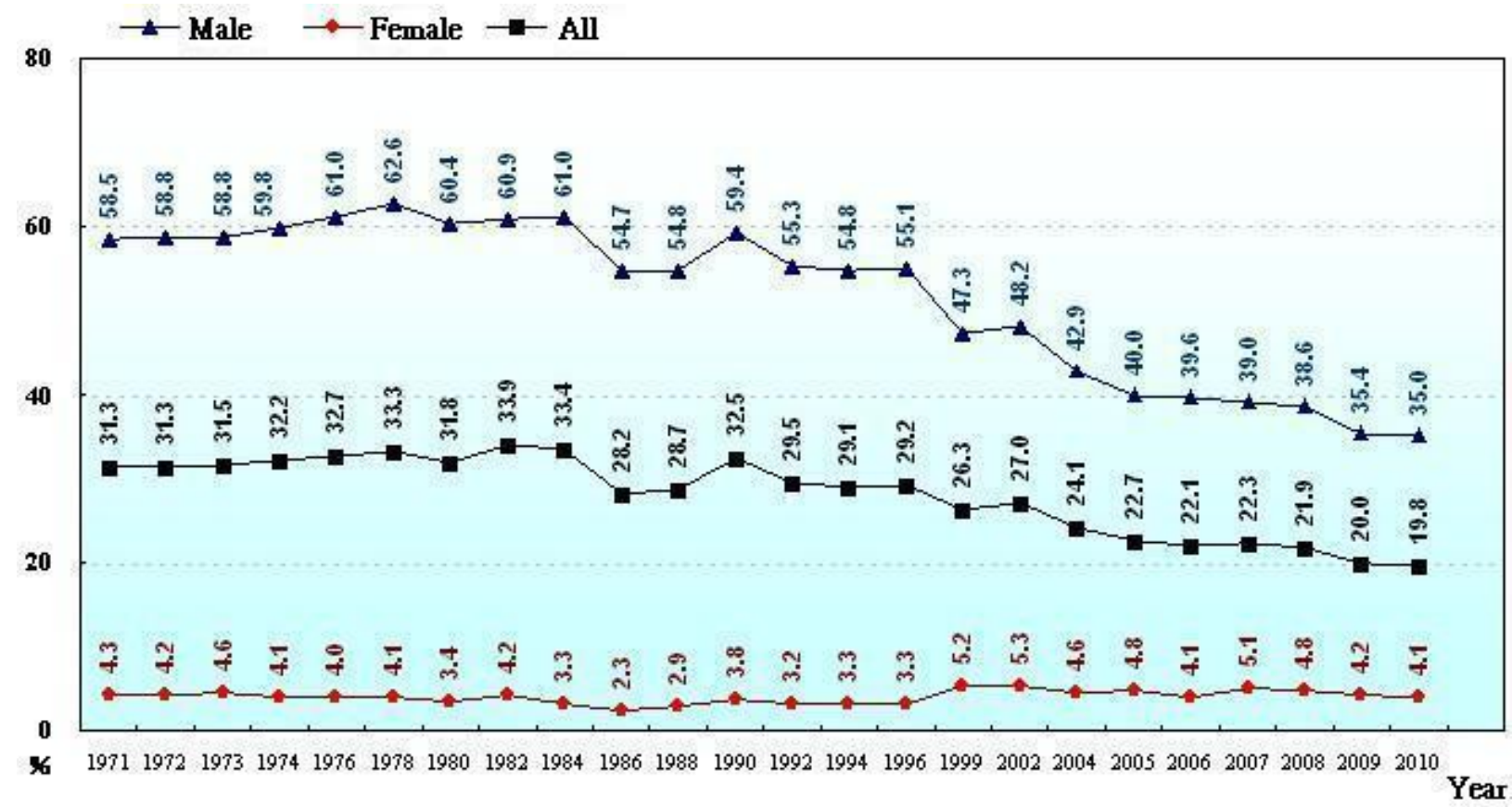

Figure E1. Smoking rate among people age 18 or more in Taiwan (downloaded from http://tobacco.hpa.gov.tw/Show.aspx?MenuId=581, the Health Promotion Administration, Ministry of Health and Welfare, Taiwan, on August 15, 2013). 


\section{APPENDIX F.}

a. FIBC

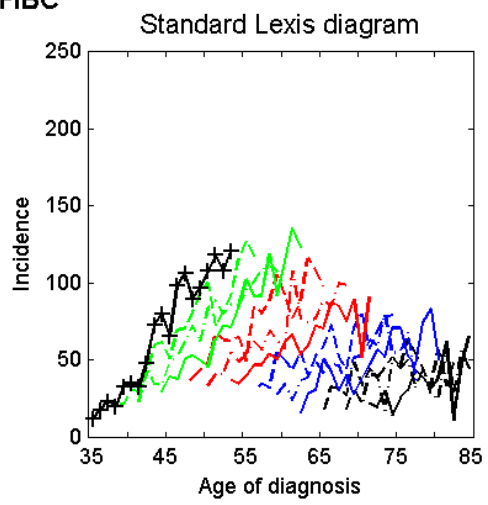

c. MADC

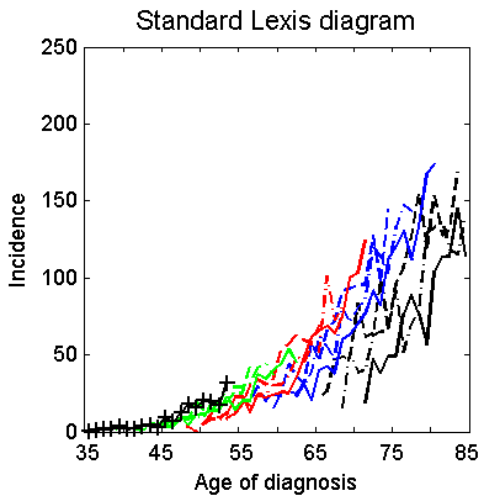

b. FADC

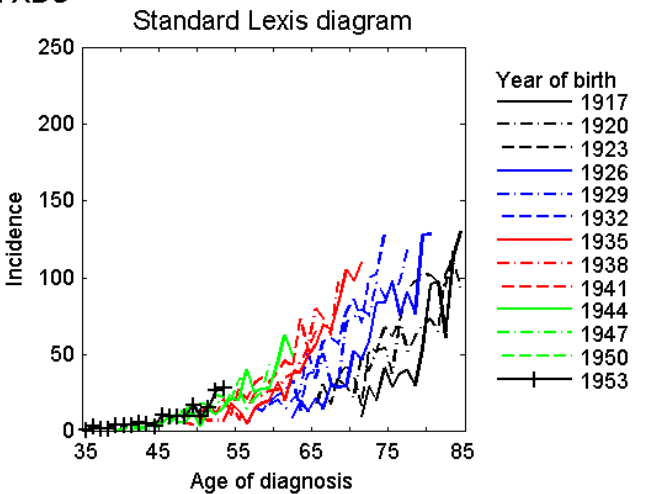

d. MSCC

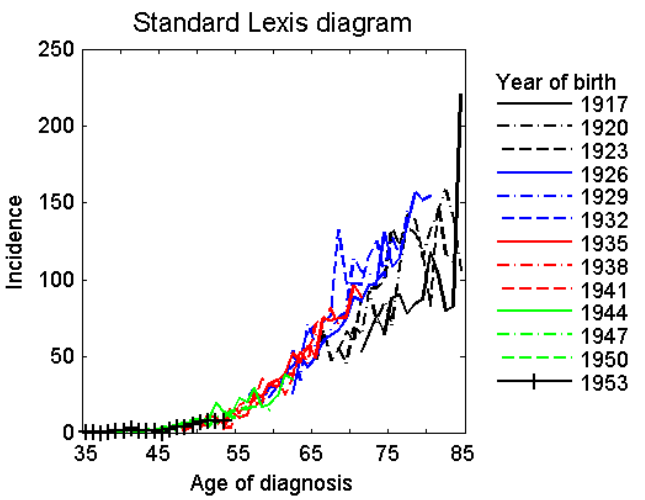

Figure F1-1. Age-specific rates by year of birth based on 1-year tabulated standard Lexis diagrams (rates versus age of diagnosis, observations within each birth-cohort are connected). 
a. FIBC

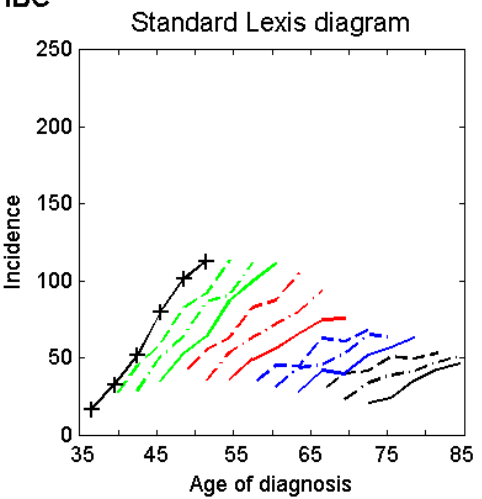

c. MADC

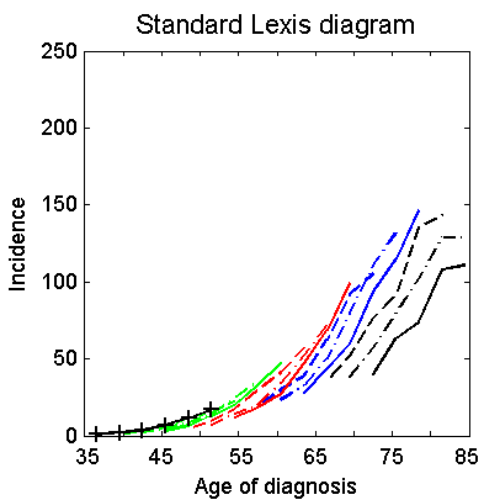

b. FADC
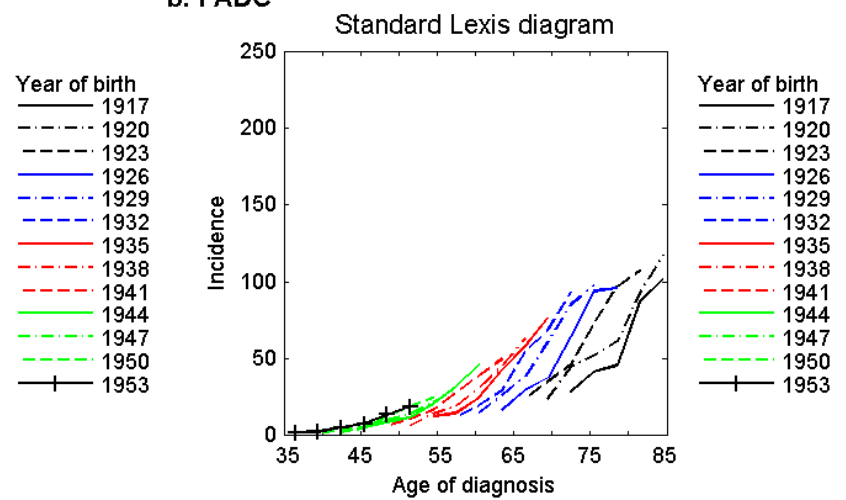

d. MSCC

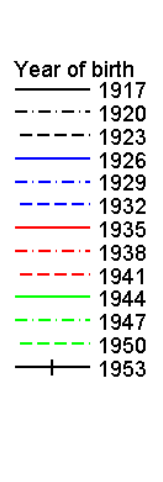

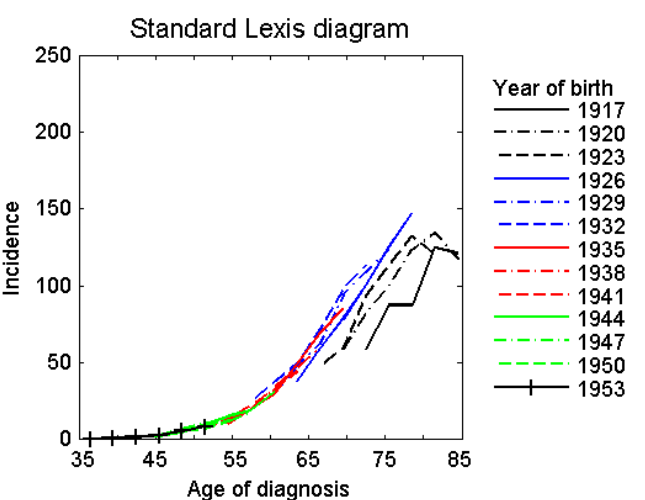

Figure F1-3. Age-specific rates by year of birth based on 3-year tabulated standard Lexis diagrams (rates versus age of diagnosis, observations within each birth-cohort are connected). 
a. FIBC

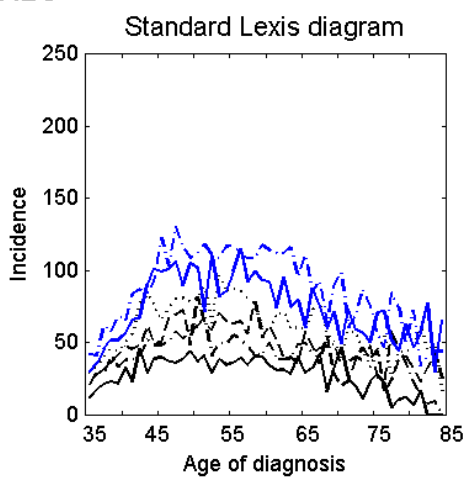

c. MADC

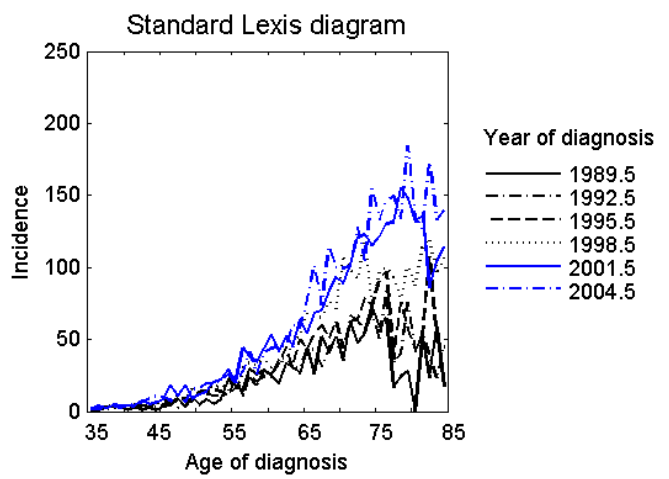

b. FADC

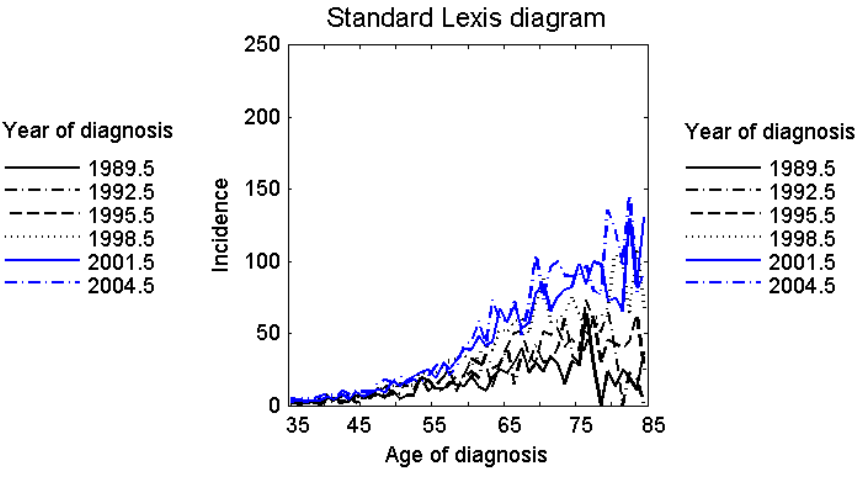

d. MSCC

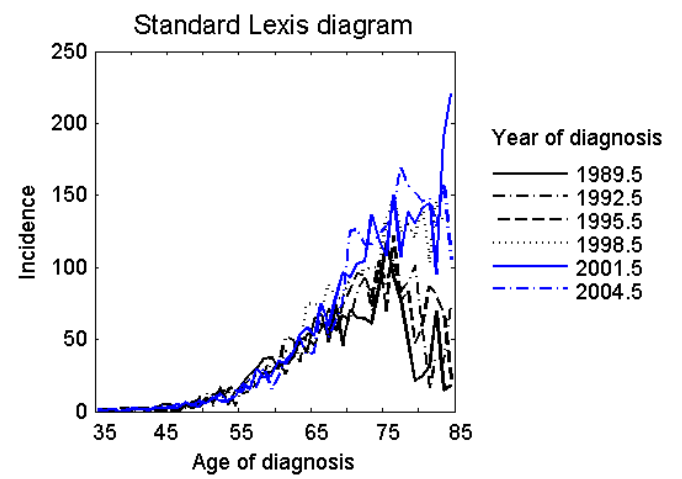

Figure F2-1. Age-specific rates by year of diagnosis based on 1-year tabulated standard Lexis diagrams (rates versus age of diagnosis, observations within each year are connected). 
a. FIBC

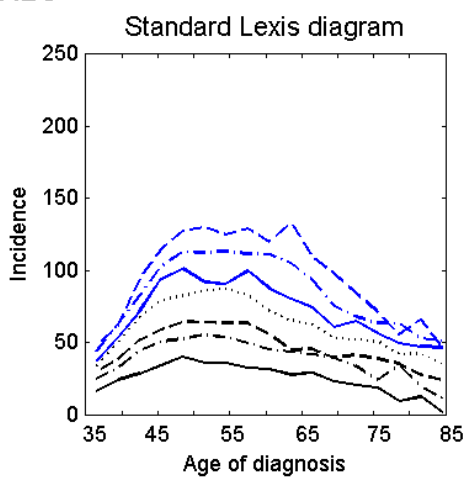

c. MADC

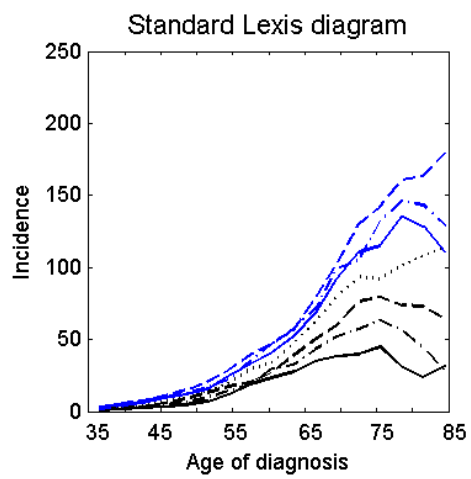

b. FADC

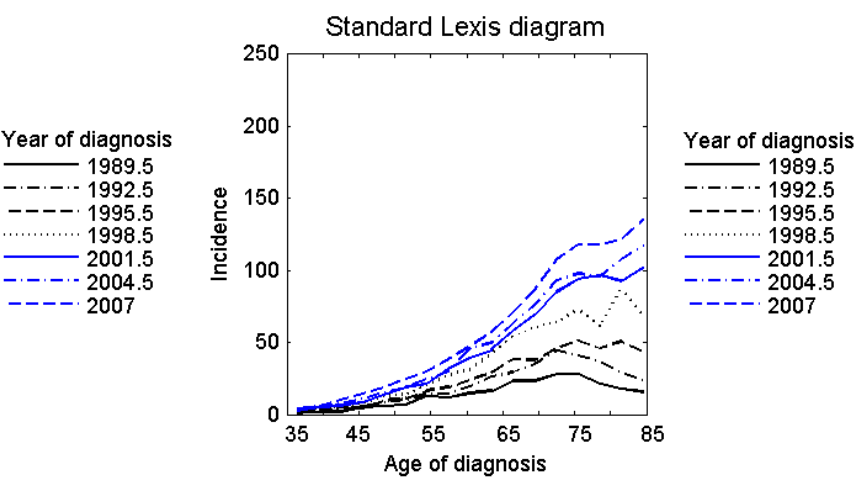

d. MSCC
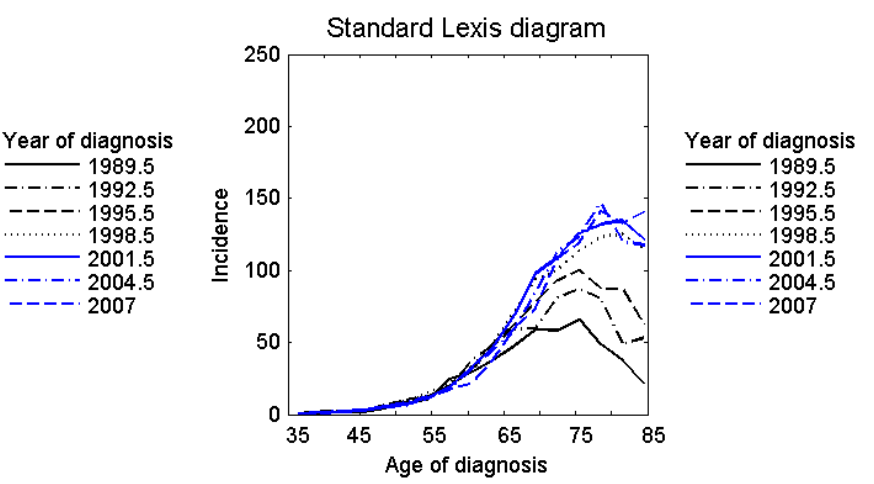

Figure F2-3. Age-specific rates by year of diagnosis based on 3-year tabulated standard Lexis diagrams (rates versus age of diagnosis, observations within each year are connected). 
a. FIBC

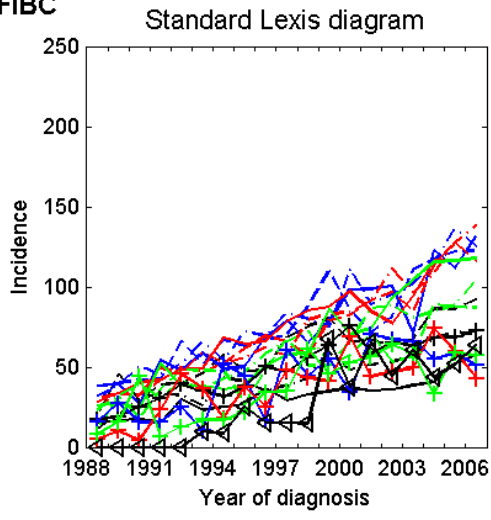

c. MADC

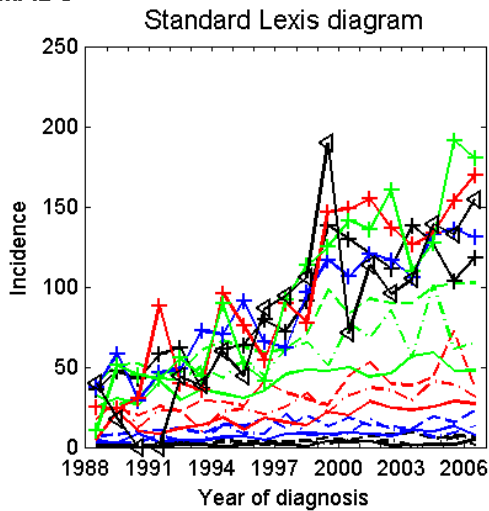

b. FADC
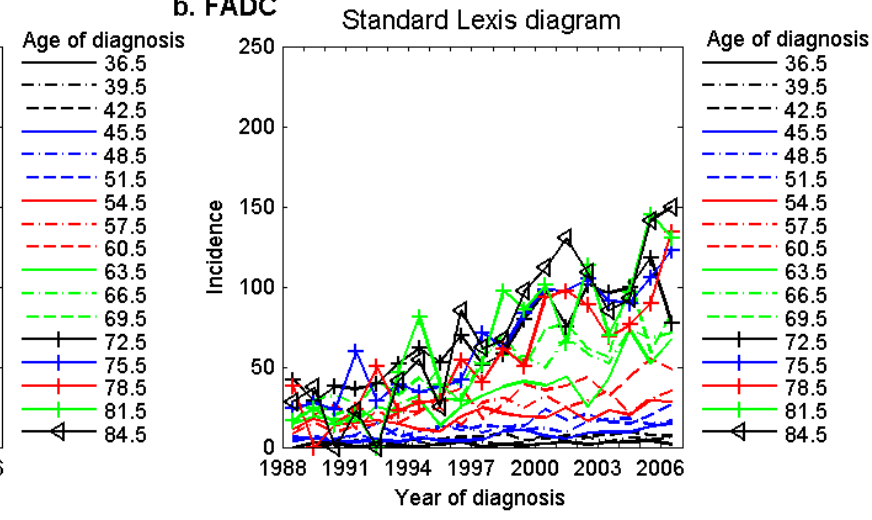

d. MSCC

Age of diagnosis

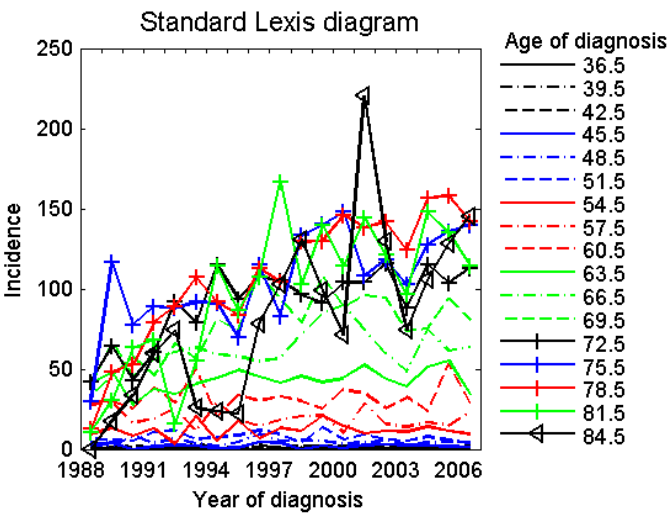

Figure F3-1. Year-specific rates by age of diagnosis based on 1-year tabulated standard Lexis diagrams (rates versus year of diagnosis, observations within each age group are connected). 
a. FIBC

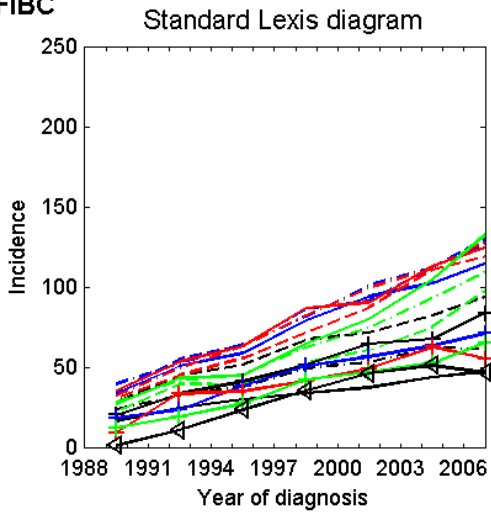

c. MADC

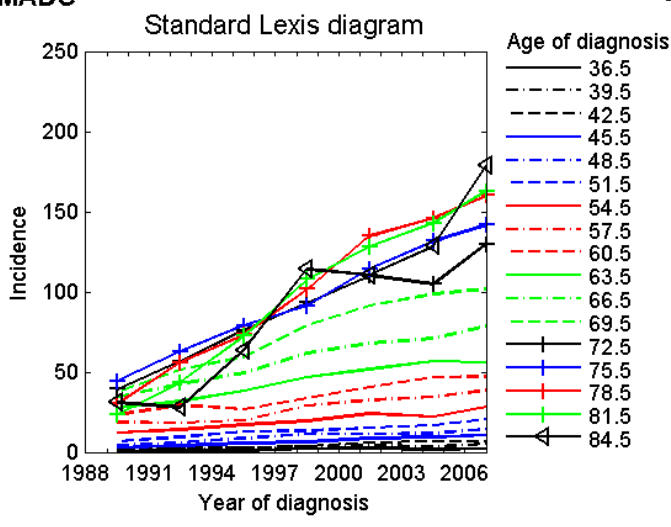

b. FADC Standard Lexis diagram

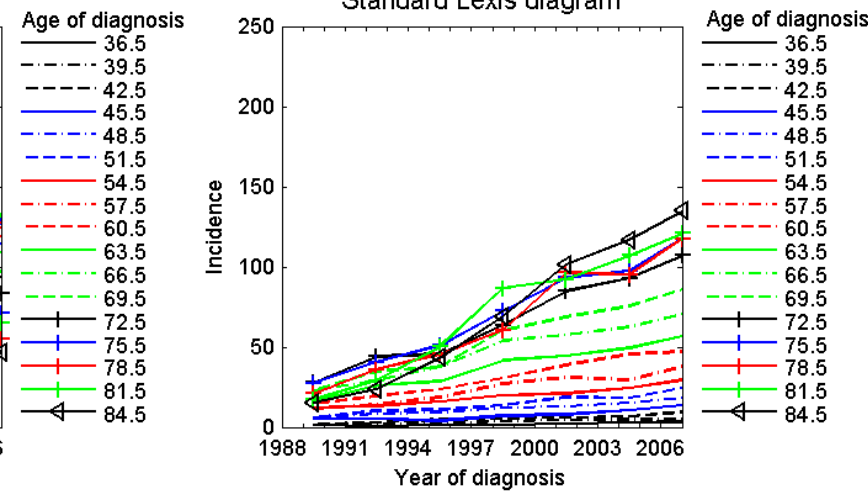

d. MSCC

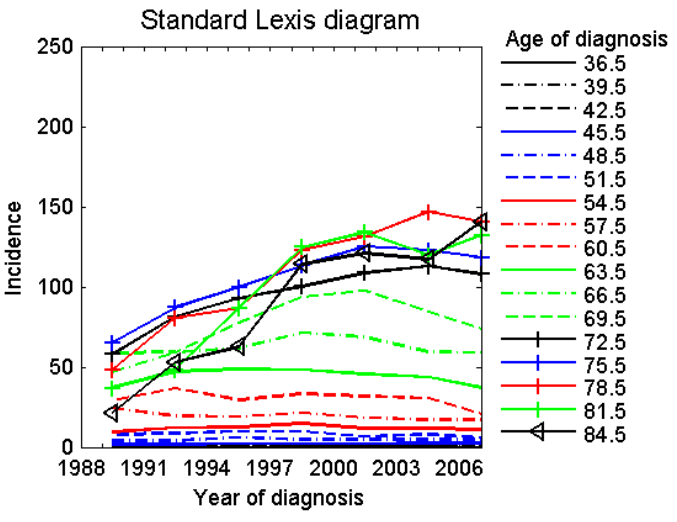

Figure F3-3. Year-specific rates by age of diagnosis based on 3-year tabulated standard Lexis diagrams (rates versus year of diagnosis, observations within each age group are connected). 

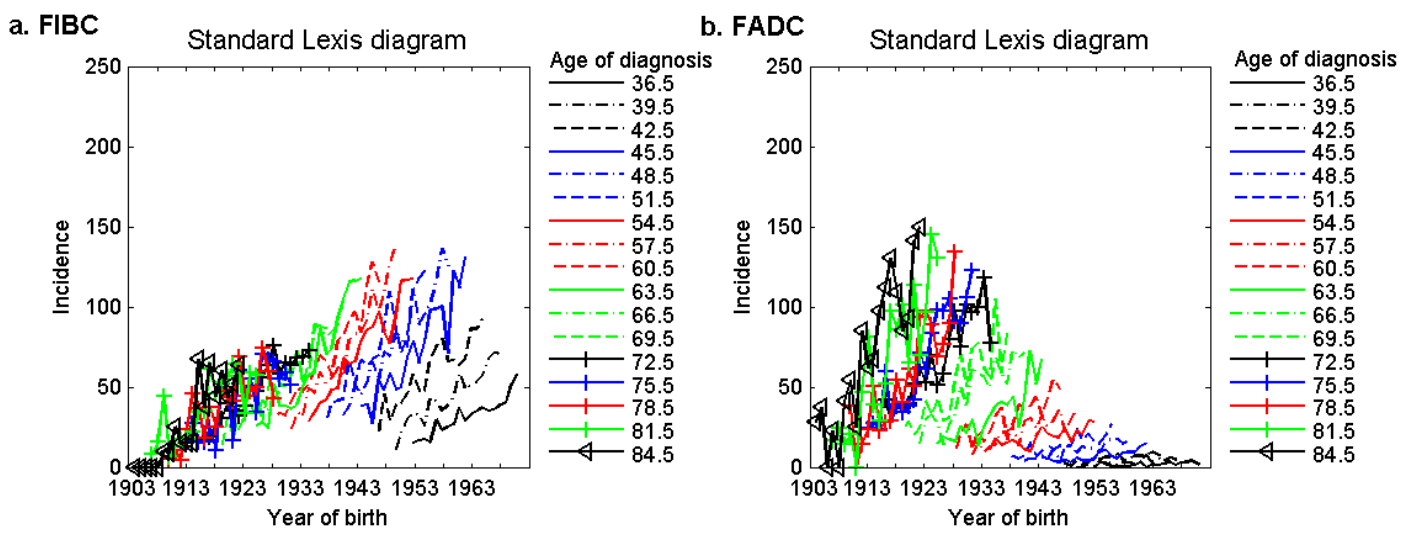

c. MADC

d. MSCC
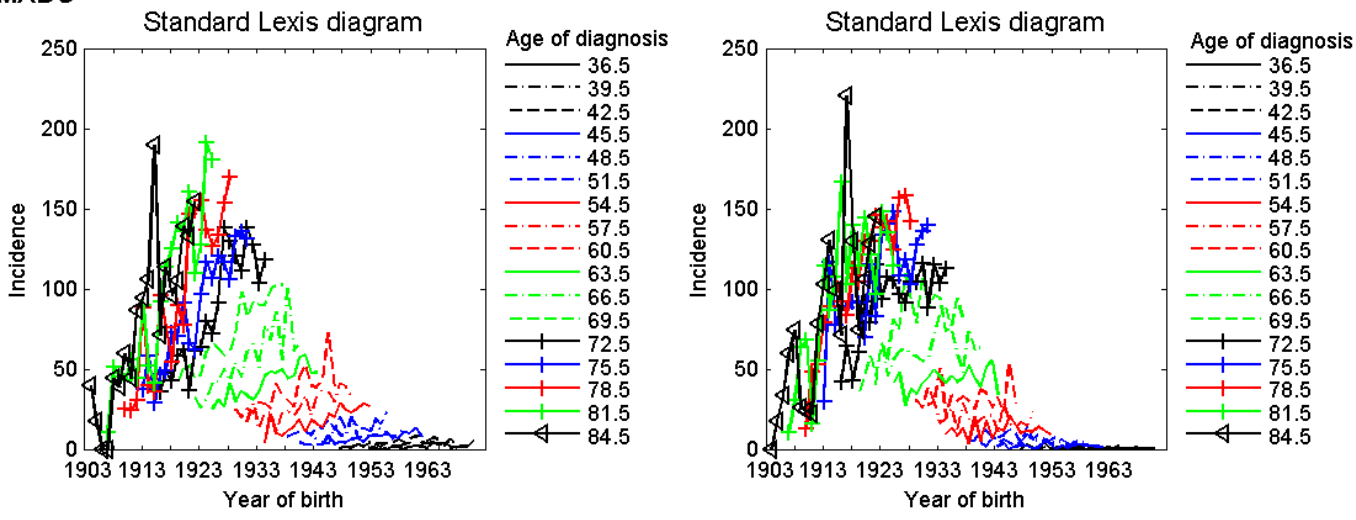

Figure F4-1. Cohort-specific rates by age of diagnosis based on 1-year tabulated standard Lexis diagrams (rates versus birth-cohort, observations within each age group are connected). 
a. FIBC

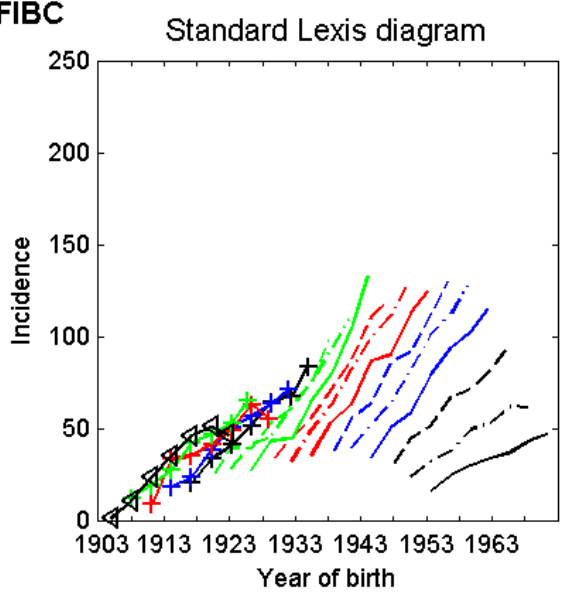

c. MADC

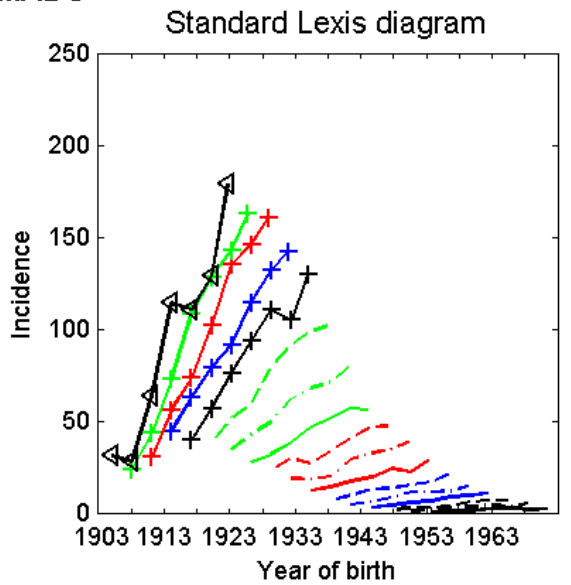

b. FADC
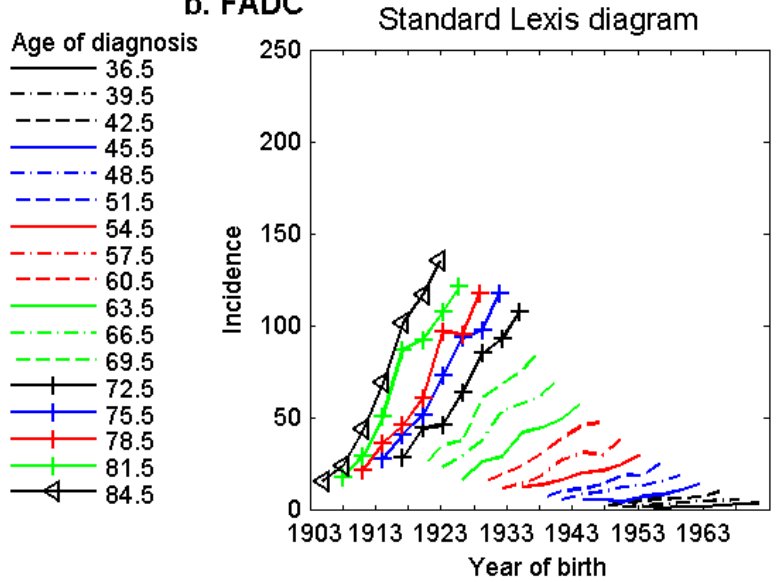

Age of diagnosis

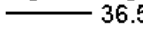

-.-. 39.5

$\begin{array}{r}---4.5 \\ \hline\end{array}$

$-\cdot-\cdot 48.5$

$----51.5$

54.5

$---0.60 .5$

63.5

-

$\longrightarrow 72.5$

+
$\longrightarrow$ 75.5 $\begin{array}{r}+ \\ +\quad 81.5 \\ \hline\end{array}$ $-4-84.5$

d. MSCC

Age of diagnosis

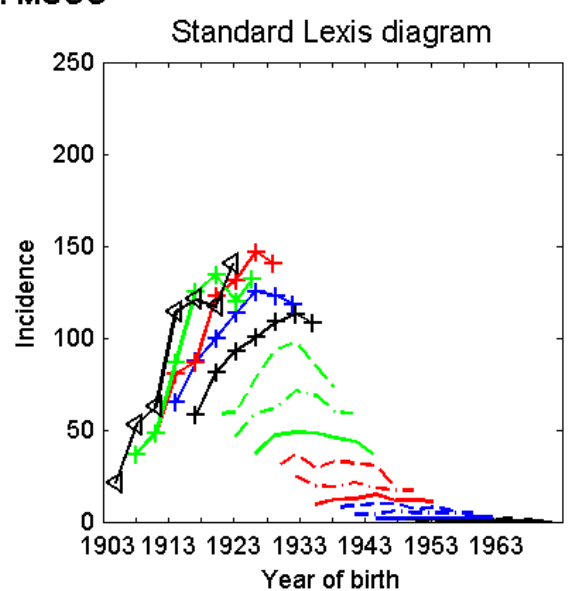

Age of diagnosis 36.5 $----42.5$ 45.5 $--.-48.5$ $----51.5$ 54.5 $-\cdot-\cdot 57.5$ $----60.5$ 63.5 - 66.5 $\longrightarrow 72.5$ $+75.5$ \begin{tabular}{r}
1 \\
\hdashline-81.5 \\
-484.5
\end{tabular}

Figure F4-3. Cohort-specific rates by age of diagnosis based on 3-year tabulated standard Lexis diagrams (rates versus birth-cohort, observations within each age group are connected). 